\title{
Route entropy based capacity reliability assessment and application in multi-objective satisfactory optimization of logistics network
}

\author{
Xin Miao ${ }^{1 \star}$, Bao Xi ${ }^{2}$, Ming Guan ${ }^{3}$ and Yan-hong Tang ${ }^{1}$ \\ ${ }^{1}$ School of Management, Harbin Institute of Technology, Harbin 150001, People's Republic of China. \\ ${ }^{2}$ School of Public Administration, Dalian University of Technology, Dalian 116024, People's Republic of China. \\ ${ }^{3}$ School of Economics and Management, Xuchang University, Xuchang 461000, People's Republic of China.
}

Accepted 19 January, 2011

\begin{abstract}
Capacity reliability assessment and application are emerging active area in transportation science. This paper built assessment model of capacity reliability of road network from the perspective of route entropy by referring to relevant concepts and methods applied in water distribution system. Route entropy represents the uncertainty of route selection of macro traffic flow in a road network. The probability space and calculative model of route entropy were introduced as a new method with regard to the field of capacity reliability research in transportation science. This paper further demonstrated a feasible method for applying resultant capacity reliability in logistics network optimization. The decision of how to optimize a logistics network depends upon several criteria that are sometimes conflicting under a set of constraints. This paper analyzed and modeled the conflicting objectives through forming satisfactory function. The proposed approach captured essential requirements of actual logistics network and considered them synthetically into unified dimension for optimization. Improved genetic algorithm was applied to search solutions, and the applicability and validity of the method were further demonstrated by a numerical example. This paper contributed to the development of capacity reliability theory in transportation science. It may bring some inspiration to relevant scientific researchers and engineers.
\end{abstract}

Key words: Road traffic, road network, traffic flow, route choice, entropy, uncertainty, multi-objective, satisfactory optimization, logistics network

\section{INTRODUCTION}

It is well acknowledged that traffic congestion is a severe problem faced by the development of modern urban civilization (Akgungor and Dogan, 2009; Gundogdu, 2009; Tang and Xi, 2010). Emerging techniques and policies may solve this problem, but before it is solved, people have to find ways to assess capacity reliability of road traffic network so that they can distinguish where the vulnerable parts are and provide support for designing

*Corresponding author. E-mail: miaoxin@yahoo.cn or miaoxin@hit.edu.cn.

concrete countermeasures.
Logistics network optimization has to meet several goals simultaneously and an appropriate logistics distribution scheme involves non-economic factors. Operation at absolute minimum cost can no longer be the only criterion for logistics distribution due to increasing concern for service level and reliability (Gursoy, 2010; Kofteci et al., 2010). However, how to measure reliability and apply it in logistics optimization is still a difficult problem to be solved. The purpose of multi-objective satisfactory optimization for logistics network is to generate efficient alternatives, which can then be presented to the decision-maker so that he/she can $3336 \quad$ Sci. Res. Essays 
select the preferred alternative while considering conflicting and non-commensurate objectives (Malakooti, 1989).

Multi-objective satisfactory optimization is to optimize simultaneously two or more conflicting objectives under a set of constraints (Liu et al., 2000). General mathematical form of a multi-objective satisfactory optimization problem is formulated as follows:

$$
\begin{aligned}
& \text { Minimize }\left\{f_{1}(x)=z_{1}, f_{2}(x)=z_{2}, \cdots, f_{n}(x)=z_{n}\right\} \\
& \text { Subject to } \quad x \in \Omega
\end{aligned}
$$

Where, solution $x$ is a vector of discrete decision variables, $\Omega$ is the finite set of feasible solutions. The image of a solution $x \in \Omega$ is the point $z=f(x)$ in the objective space. A point $z$ dominates $z^{\prime}$, if $z_{j}=f_{j}(x) \leq z_{j}^{\prime}=f_{j}\left(x^{\prime}\right), \forall j$ and $z_{j} \leq z_{j}^{\prime}$ for at least one $j$. A solution $x$ dominates $x^{\prime}$ if the image of $x$ dominates the image of $x^{\prime}$. A solution $x^{*} \in \Omega$ is a nondominated (or efficient) solution if there is no $x \in \Omega$ such that $z=f(x)$ dominates $z^{*}=f\left(x^{*}\right)$. The solutions that are non-optimal solutions within the entire search space are named as Pareto-optimal solutions and constitute the Pareto-optimal set or Pareto-optimal frontier (Li, 2009).

Satisfactory optimization ( $\mathrm{Li}$ and $\mathrm{Hu}, 2007$ ) extends optimization theory through replacing optimal solution with satisfactory solutions. It can properly consider satisfactory design of system performance indexes and optimization of system parameters, and at the same time, take into account constraints and objective function integrally (Liu, 2006). This paper tries to achieve a satisfactory logistics distribution network and the basic factors considered are transportation cost, average delay and capacity reliability obtained through the route entropy method.

\section{LITERATURE REVIEW}

The concept of capacity reliability was first put forward by Chen et al. (1999) and was defined as the probability that the network can accommodate a certain traffic demand within a predefined service level. Later, Chen et al. (2002) extended the capacity reliability analysis by presenting comprehensive methodology, which related capacity reliability with uncertainty analysis. Sumalee and Kurauchi (2006) presented a Monte-Carlo simulation method to estimate capacity reliability. da Silva et al. (2010) also put forward a Monte Carlo simulation approach based on cross-entropy method to evaluate generating capacity reliability. There is no doubt it would be better to incorporate uncertainty into capacity reliability analysis as the concept in itself is unification of a fuzzy and random meaning in a dynamic environment, while fuzziness and randomicity are typical expression of uncertainty (Miao et al., 2009). Castans (1962) discovered that there are logarithmic relation between entropy and uncertainty. Later, entropy was applied in assessment of uncertainty in hydrologic systems (Amorocho and Espildor, 1973), uncertainty relations of wave mechanics (Bialynickibirula and Mycielski, 1975), uncertainty estimation of power spectrum (Schott and Mcclellan, 1984), uncertainty in past lifetime distributions (Di Crescenzo and Longobardi, 2002), uncertainty of resource allocation (Johansson and Sternad, 2005) and so on.

Entropy has been applied to assess reliability with uncertainty for water distribution networks and Awumah et al. $(1990,1991)$ were the first to suggest the use of Shannon's entropy (Shannon, 1948) for measuring the reliability of water distribution networks. Tanyimboh and Templeman (1993a) were the pioneers who put forward the entropy function for application in water distribution networks. They also designed a sequential algorithm to search the distribution of maximum-entropy flow for single-source networks (Tanyimboh and Templeman, 1993b), and in their paper, the only known conditions are the network topology, the flow directions in each pipe, and the supplies and demands at each node. Further, they demonstrated that there is intrinsic relationship between the entropy and reliability of water distribution networks (Tanyimboh and Templeman, 2000), and what they disclosed was in accordance with the maximum entropy principles (Rajagopal and Teitler, 1989), that is, a network will be more reliable if it possesses higher entropy. Ang and Jowitt (2005a) put forward a named path entropy method to calculate entropy of water distribution networks, and later they further develop this method into entropy calculation for multiple-source water distribution networks (Ang and Jowitt, 2005b).

As we know, traffic flow theory can track its origin to analogy of hydrokinetics. British scholars Lighthill and Whitham (1955) made an analogy to consider traffic flow as liquid. Through studies of traffic flow on long congested roads, they put forward the theory of traffic kinetic waves, which adopted basic theory of hydrodynamics to analyze the movement of traffic flow and obtained the continuous equation for traffic flow study (Miao et al., 2008). Therefore, we believe that entropy can be adopted to analyze the capacity reliability of road traffic network by referring to the methods used for water distribution networks.

For logistics network optimization, although network analysis and optimization are widely studied in the operations research literature (Jeet et al., 2009), traditional models that focused on minimizing fixed costs of facility location and transportation costs are no longer suitable for logistics network optimization (Eskigun et al., 2005). Recent years have seen an increasing concern on this aspect, such as Barnhart and Shen (2005), Cordeau 
et al. (2006), Candas and Kutanoglu (2007), Cheong et al. (2007), Cordeau et al. (2008), Ho and Emrouznejad (2009), Jeet et al. (2009), Chiou (2009), etc. Generally speaking, the superiority of these works over the previous is that they considered multi-objective in design and optimization process, and adopted heuristic or improved search algorithms for problem solving. However, these works fall into the category of optimal optimization.

Compared with fuzzy multi-criteria optimization (Sreekumar and Mahapatra, 2009), satisfactory optimization does not involve fuzzy disposal and complicated computation that makes it easier for clear expression of manager's preference, and hence, is efficient in identifying potential solutions. If a multiobjective satisfactory optimization problem is well formulated, there should more than one solution that simultaneously leads each objective to its fullest, and a set of compromising solutions will be provided for selection. The use of satisfactory optimization allows managers to consider many worthwhile measures of performance of a system and to identify good scheme alternatives and the trade-offs between them (Badri et al., 1998). Accordingly, satisfactory optimization method is convenient for practical application. As we know, "real world optimization problems deal with competing incommensurable objectives in a complex search space. Improvement in one objective often leads to degradation in at least one of the remaining objective functions" (Ok et al., 2009). While the satisfactory optimization method "guides the multi-point searching process toward a uniformly spread-out Pareto-optimal front in multidimensional objective space. Thus, the distribution of the Pareto-optimal solutions well describes the relative importance between the multiple objectives or how the multiple objectives are competent with each other in the objective space" (Ok et al., 2009).

\section{ROUTE ENTROPY BASED CAPACITY RELIABILITY ASSESSMENT}

Referring to and distinguishing from path entropy, we call the entropy, which is applied to assess capacity reliability of road traffic network and analyze uncertainty of route choice of macro traffic flow, as route entropy.

Firstly, we need to express Shannon entropy as formula (2):

$$
S=-K \sum_{i=1}^{N} p_{i} \ln p_{i}
$$

Where, $K$ denotes a positive constant, and $p_{i}(i=1, \ldots$, $M$ denote a finite probability space, which include a series probabilistic event of finitude incompatible Miao et al. $\quad 3337$ probabilistic events.

The maximum-entropy formalism (Jaynes, 1957) is used to discover the probability distribution that leads to the highest value of uncertainty, thereby assuring that no information is inadvertently assumed. It is a property of the above entropy formula that it has its maximum value when all probabilities are equal.

The entropy to measure the uncertainty of route choice of macroscopic traffic flow is called route entropy. Traffic flow usually chooses the route with the minimum traffic impedance, and the uncertainty of route selection of macroscopic traffic flow reflects the dynamic changes of traffic impedances in different parts of the road network. Based on the principle of maximum entropy, the state corresponding to the maximum entropy has the highest reliability, and each route has the same chance to be selected and traffic flow distributed soundly in the whole road network in this state.

The ratio of the traffic volume in each route to the total traffic volume is called Route Flow Ratio (RFR) of each route. The RFR reflects the dynamicity of route choice of macroscopic traffic flow. It associates impedance characteristics with flow characteristics, and constitutes the theoretical basis for state estimation of road network.

Denote the traffic volume from trip generation spot to node $j$ as $\left(f_{j}^{i}\right), i=1 ; \cdot, N$. Where, the $N$ denotes the number of routes from trip generation spot to node $j$. Let the sum of traffic volume in each route connected to node $j$ as $q_{j 0}$, that is:

$\sum_{i=1}^{N} f_{j}^{i}=q_{j 0}$

Define probability space according to RFR:

$p_{j}^{i}=\frac{f_{j}^{i}}{q_{j 0}}$

According to formula (2), the route entropy of node $j$ can be denoted as:

$S_{j}=-\sum_{i=1}^{N} \frac{f_{j}^{i}}{q_{j 0}} \ln \frac{f_{j}^{i}}{q_{j 0}}$

Rearrange formula (5) and obtain another form:

$S_{j}=-\frac{1}{q_{j 0}}\left(\sum_{i=1}^{N} f_{j}^{i} \ln f_{j}^{i}-q_{j 0} \ln q_{j 0}\right)$

Let $j$ denote a random node and it has three directly $3338 \quad$ Sci. Res. Essays 
connected upper nodes $a_{1}, a_{2}$ and $a_{3}$. There are $n$ routes from trip generation spot $O$ to node $j$, and among the $n$ routes, there are $k$ coincident routes from $a_{1}$ to $j, m$ coincident routes from $a_{2}$ to $j$ and $n-m-k$ coincident routes from $a_{3}$ to $j$.

Further, let $R_{1}=\sum_{i=1}^{k} f_{j}^{i}, R_{2}=\sum_{i=k+1}^{k+m} f_{j}^{i}$ and $R_{3}=\sum_{i=k+m+1}^{n} f_{j}^{i}$, then;

$R_{1}+R_{2}+R_{3}=q_{j 0}$

Let $q_{a_{1} 0}, q_{a_{2} 0}, q_{a_{3} 0}$ denote the traffic volume on node $a_{1}$, $a_{2}$ and $a_{3}$ respectively, then

$$
\begin{aligned}
& f_{a_{1}}^{i} / q_{a_{1} 0}=f_{j}^{i} / R_{1}, \quad i \in(1, k) \\
& f_{a_{2}}^{i} / q_{a_{2} 0}=f_{j}^{i} / T_{2}, \quad i \in(k+1, k+m) \\
& f_{a_{3}}^{i} / q_{a_{3} 0}=f_{j}^{i} / R_{3}, \quad i \in(k+m+1, n)
\end{aligned}
$$

According to formula (5), get the following:

$$
\begin{aligned}
& S_{a 1}=-\sum_{i=1}^{k} \frac{f_{j}^{i}}{R_{1}} \ln \frac{f_{j}^{i}}{R_{1}}=-\frac{1}{R_{1}}\left[\sum_{i=1}^{k} f_{j}^{i} \ln f_{j}^{i}-R_{1} \ln R_{1}\right] \\
& S_{a 2}=-\sum_{i=k+1}^{k+m} \frac{f_{j}^{i}}{R_{2}} \ln \frac{f_{j}^{i}}{R_{2}}=-\frac{1}{R_{2}}\left[\sum_{i=k+1}^{k+m} f_{j}^{i} \ln f_{j}^{i}-R_{2} \ln R_{2}\right] \\
& S_{a 3}=-\sum_{i=k+m+1}^{n} \frac{f_{j}^{i}}{R_{3}} \ln \frac{f_{j}^{i}}{R_{3}}=-\frac{1}{R_{3}}\left[\sum_{i=k+m+1}^{n} f_{j}^{i} \ln f_{j}^{i}-R_{3} \ln R_{3}\right]
\end{aligned}
$$

Let $q_{a 1 j}, q_{a 2 j}$ and $q_{a 3 j}$ denote the traffic volume on road section $a_{1 j}, a_{2 j}$ and $a_{3 j}$ respectively, and let $Q_{j}$ denote the total traffic volume coming to node $j$, then

$$
R_{1} / q_{j 0}=q_{a 1 j} / Q_{j}, R_{2} / q_{j 0}=q_{a 2 j} / Q_{j}, R_{3} / q_{j 0}=q_{a 3 j} / Q_{j}
$$

The entropy of node $j$ can be calculated as:

$$
S_{j}=\frac{1}{q_{j 0}}\left[\sum_{i=1}^{k} f_{j}^{i} \ln f_{j}^{i}+\sum_{i=k+1}^{k+m} f_{j}^{i} \ln f_{j}^{i}+\sum_{i=k+m+1}^{n} f_{j}^{i} \ln f_{j}^{i}-q_{j 0} \ln q_{j 0}\right]
$$

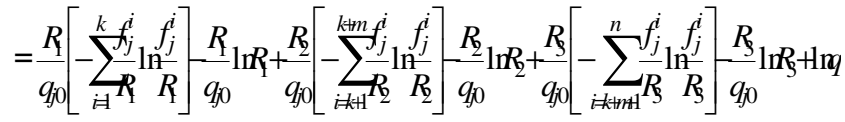

$$
\begin{aligned}
& =\frac{q_{a d j}}{Q_{j}} S_{a 1}+\frac{q_{a 2 j}}{Q_{j}} S_{a 2}+\frac{q_{a b j}}{Q_{j}} S_{a 3}-\frac{R_{1}}{q_{j 0}} \ln \frac{R_{1}}{q_{j 0}}-\frac{R_{2}}{q_{j 0}} \ln \frac{R_{2}}{q_{j 0}}-\frac{R_{3}}{q_{j 0}} \ln \frac{R_{3}}{q_{j 0}}
\end{aligned}
$$

If $j$ has $n$ upper nodes and let $p_{n j}=q_{n j} / Q_{j}=R_{n} / q_{j 0}$, then the route entropy of node $j$ can be denoted as:

$$
S_{j}=\sum_{n \in U(j)}\left(-p_{n j} \ln p_{n j}+p_{n j} S_{n}\right)
$$

Where, $U(j)$ is the set of the nodes which directly connected to node $j$. For a road network with multiple trip generation spots, the upper node of the trip generation spots can be seen as a virtual source and $S_{n}$ denotes the route entropy of the upper node $n$.

The above model can be solved by sequential algorithm (Wu and Tian, 2003). The source or virtual source of trip generation can be regarded as the initial node of a network and there is no conflux in such a node, therefore, there is zero entropy in the initial node. The initial node is the starting point of sequential algorithm, which will search all the routes of the network and easy to be adapted into computer program. The entropy of a node denotes the uncertainty of the whole process that the traffic flow start from the source and flow into this node. From formula (16), we know that the entropy at any node include not only the uncertainty of the upper nodes but also the uncertainty of conflux in current node. In a word, route entropy can reflect the uncertainty of route choice of macro traffic flow, that is, the capacity reliability of a road network.

\section{THE MULTI-OBJECTIVE OPTIMIZATION MODEL}

\section{SATISFACTORY}

A logistics network can be expressed as a weighting nondirectional graph $G=(V, E)$, where, $V$ is the node set of the network, $E$ is the link set of the distribution paths. Let $|V|$ denote the node number of $G$ and $|E|$ denote the link number. Link $\varepsilon_{i j}=\left(v_{i}, v_{j}\right)$ denotes path between node $v_{i}$ and $v_{j}$. If $\varepsilon_{i j}=0$, it means there is no path between the two nodes, where, $i, j=1,2, \ldots, n$ and $v_{i}, v_{j} \in V$.

To express state of each distribution path by the above graph, let $R_{+}$denote positive real number set and $R^{+}$ denote non-negative real number set, then we have distribution delay delay $\left(e_{i j}\right): E \rightarrow R_{+}$, transportation cost 


$$
\begin{aligned}
& \operatorname{cost}\left(e_{i j}\right): E \rightarrow R_{+} \quad \text { and capacity } \\
& \operatorname{rel}\left(e_{i j}\right): E \rightarrow R^{+} \text {. } \\
& \text { Known conditions: } \\
& \text { 1) Matrix of freight vehicles between two nodes } \\
& \text { 2) Road capacity values } \\
& \text { 3) Function of transportation cost } \\
& \text { 4) Reliability probability of road section and nodes }
\end{aligned}
$$

According to the above network, multi-objective satisfactory optimization model for logistics network design can be expressed as:

$$
\begin{aligned}
& \text { (Minimize }(G), D(G), 1-R(G) \\
& \text { Maximiz@verall_satG })=F(\text { Sat_d }(G), \text { Sat_d }(G), \text { Sat_r }(G)) \\
& \left\{S_{\perp} d G\right)=g(C(G)) \\
& \text { Sat_d }(G)=h(D(G)) \\
& \text { Sat_r } r(G)=s(R(G))
\end{aligned}
$$

\section{Constraints:}

(1) Reliability and practical existence of road network

(2) Capacity range of road network

Where, $C(G)$ denotes transportation cost in the network, $D(G)$ denotes average distribution delay and $R(G)$ denotes reliability of the network. Sat_c(G), Sat_d(G) and Sat_r $(G)$ denote satisfaction functions of corresponding performance indexes, that is, cost, delay and reliability, respectively. Overall_sat $(G)$ denotes overall satisfaction function. Minimization of $C(G), D(G)$ and $1-R(G)$ denote minimization of transportation cost, average distribution delay and unreliability of distribution network, respectively. Maximization of Overall_sat(G) denotes maximization of overall satisfaction degree of the logistics network $G$.

Satisfaction function is used for quality evaluation of satisfactory solutions (Huang et al., 2008). In practice, different satisfaction functions of performance indexes should be designed according to different backgrounds of optimization problems.

Overall satisfaction function is to transform multiobjective optimization model into single objective optimization model by synthesizing two or more performance indexes together. Here, a frequently used overall satisfaction function, which has simple structure, is adopted in this paper.

$$
S w=f(s)=\sum_{i=1}^{m} \omega_{i} s_{i}, \sum_{i=1}^{m} \omega_{i}=1
$$

Where, $w_{i}$ is the weight of $s_{i}, i=1,2, \ldots, m$. By formula

$$
\text { Miao et al. } \quad 3339
$$

(18), overall satisfaction function $S w$ can be obtained from linear weighting computation of $s_{i}$. Weight $w_{i}$ reflects manager's preference for performance index $s_{i}$. Analytical hierarchy process can be applied to determine $w_{i}$.

\section{METHOD TO SOLVE THE MODEL}

Logistics network consists of large number nodes, complicated connections and different logistics volumes. Therefore, as for optimization of logistics network, transportation cost, logistics volumes, distribution routes, node location, node capacity, distribution delay and other factors should be taken into integrated consideration to achieve low-cost, high-performance network. A feasible approach for network optimization is to divide a whole network into several subnetworks and optimize these subnetworks in advance, and then, consider each subnetwork as a node to design the overall network structure. Accordingly, optimization of logistics network may follow the following steps:

1) Divide Logistics network into subnetworks;

2) Optimize the connections of nodes and links in each subnetwork;

3) Optimize the connections among subnetworks.

Here, improved genetic algorithm is applied to search the satisfactory solution of logistics network optimization problem, and the objective is to find a network that has merits of high reliability, low cost and less delay. Since these indexes have different dimensions, overall satisfaction function is applied to integrate the three indexes into one dimension.

\section{Subnetwork division}

The principle to be followed in subnetwork division process is that the logistics volume between any two subnetworks should be kept as small as possible and the logistics volume in each subnetwork should be kept as large as possible. In other words, two nodes with less logistics volume between them should be assigned to different subnetworks and two nodes with large logistics volume between them should be assigned to one subnetwork to enhance efficiency and cohesion of a subnetwork, and at the same time, this approach can cut down transportation cost and distribution delay between any two subnetworks.

\section{Simplification of the network}

Assume there are $n$ distribution centers and $m$ subcenters in logistics network. Figure 1 shows a simplified logistics network with 4 distribution centers and 8 subcenters.

Freight volume between subcenters can be denoted by a $m \times m$ -matrix $U$, thereinto, $u_{i j}$ denotes freight volume from subcenter $i$ to subcenter $j$. Let a $n \times n$-matrix $X_{1}$ denote the connection relationship among distribution centers. Thereinto,

$x_{1 i j}= \begin{cases}1 & \text { if } i \text { connectwith } j \\ 0 & \text { else }\end{cases}$

Let a $n \times m$-matrix $X_{2}$ denote the affiliation of subcenters to $3340 \quad$ Sci. Res. Essays 


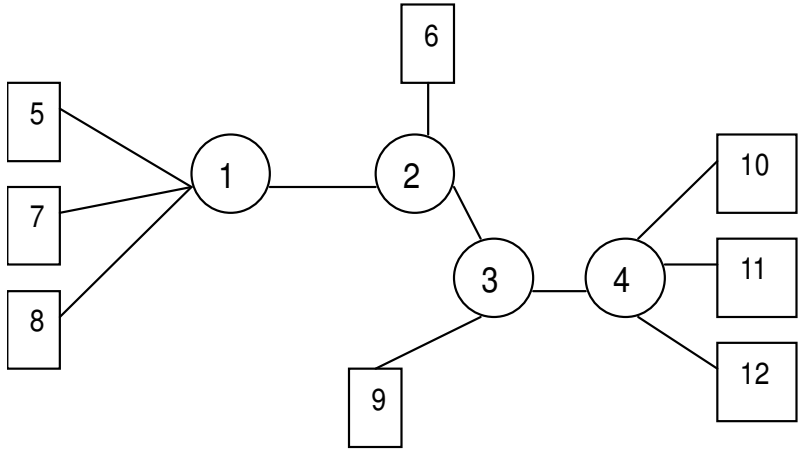

Distribution center

Distribution subcenter

Figure 1. Simplified logistics network.

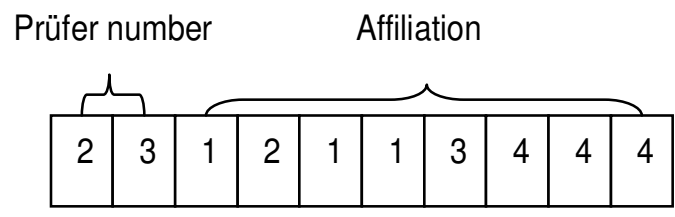

Figure 2. Code of distribution centers and subcenters.

distribution centers. Thereinto,

$x_{2 i j}= \begin{cases}1 & \text { if subcenter } j \text { affiliates to center } i \\ 0 & \text { else }\end{cases}$

Then, $\sum_{i=1}^{n} x_{2 i j}=1 \quad \forall j=1,2, \cdots, m$. Let a $n \times n$-matrix $T$ denote freight volume among distribution centers, thereinto, $t_{i j}$ denotes freight volume from a subcenter, which affiliates to center $i$, to center $j$. Then, $T=X_{2} U X_{2}^{T}$, where, $X_{2}^{T}$ is the transposed matrix of $X_{2}$.

\section{Satisfactory optimization algorithm}

For real-world multi-objective problems, the evaluation of objective functions usually requires a large amount of computation. Moreover, solving multiobjective problems often requires much longer computing time than solving single-objective problems as a result of multi-dimension to be considered for optimization. Therefore, it is essential to design efficient algorithm for solving multi-objective problems. This paper uses improved GA to speed up search process.

\section{Code scheme}

Use Prüfer code for distribution centers and clustering code for subcenters.

\section{Prüfer code:}

Setp 1: assume node $i$ as the minimal labelled node in tree $T$; Setp 2: if node $j$ is the adjacent node of $i$, let the label of $j$ as the $i^{\text {th }}$ iterm of the Prüfer sequence;

Setp 3: delete node $i$ and link $(i, \jmath)$, and let $n=n-1$;

Setp 4: repeat the above steps until only two nodes remained, then a sequence with $n-2$ number can be obtained.

\section{Reductive algorithm:}

Prüfer sequence can be reverted to a tree. Assume a Prüfer sequence consists of $n-2$ numbers, and the reductive algorithm is shown as follows:

Setp 1: Assume $W$ as a universal set of nodes, $W=\{1,2, \ldots n\} . P$ is a Prüfer sequence, $\bar{P}$ is the complementary set of $P$;

Setp 2: Assume $i$ is the minimal labelled legal node in $\bar{P}$ and $j$ is the leftmost number in $P$, then add link $(i, j)$ into tree $T$, and delete $i$ and $j$ from $P$ and $\bar{P}$ respectively; judge whether $j$ exist in $P$, if it does not exist, add $j$ into $\bar{P}$, and repeat step 2 until $P$ is empty;

Setp 3: If there is no number in $P, \bar{P}$ must have two nodes $r$ and $s$, then add link $(r, s)$ into tree $T$, end.

Prüfer sequence is applicable to code a tree. Distribution centers can be denoted by Prüfer sequence with $n-2$ numbers. In order to express the relation of distribution centers and subcenters, subcenters can be denoted by clustering code. For $n$ distribution subcenters and $m$ distribution centers, the chromosome consists of $n+m-2$ numbers. For example, the code for Figure 1 is shown in Figure 2, thereinto, the first two numbers are Prüfer numbers, and the rest eight numbers respectively denote the $i^{\text {th }}$ subcenter affiliated to one of the distribution center $1,2,3$, or 4 .

\section{Evaluation function}

Three performance indexes, that is, transportation cost, average delay and capacity reliability are the main consideration and their satisfaction functions need to be determined. Then determine weights for each performance index to constitute overall satisfaction function.

\section{Satisfaction function of performance indexes:}

Satisfaction functions of the three performance indexes can be expressed as Figures 3 to 5 (Wen et al., 2006):

\section{Overall satisfaction function:}

Overall satisfaction function corresponding to the above satisfaction functions of performance indexes is constituted as formula (21):

Overad $d(x)=\frac{w_{c} \operatorname{satd}(x)+w_{d} \operatorname{sat} d(x)+w_{r} \operatorname{sat} \underline{t}(x)}{w_{c}+w_{d}+w_{r}}$

Where, $w_{c}, w_{d}$ and $w_{r}$ are the weights of satisfaction degree of performance indexes. 


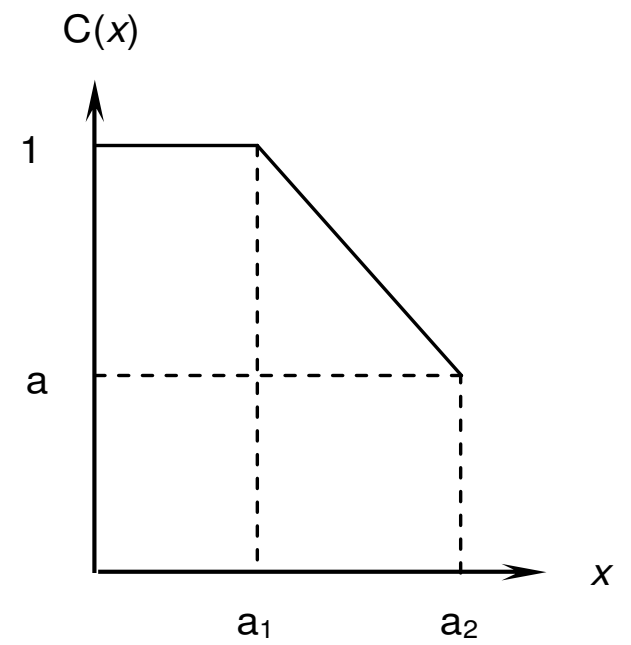

Figure 3. Satisfaction function of transportation cost.

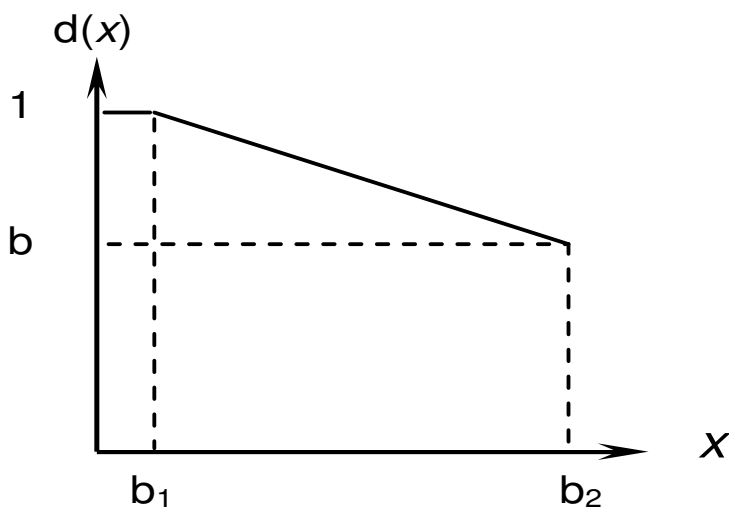

Figure 4. Satisfaction function of average delay.

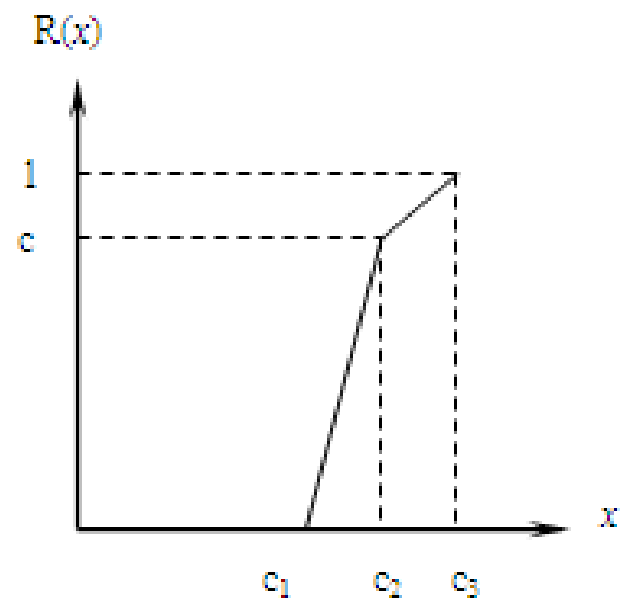

Figure 5. Satisfaction function of capacity reliability.

Miao et al.

\section{Genetic manipulation}

Selection, crossover and mutation are implemented as previously mentioned. Roulette rules can be applied in selection process of logistics network. In order to ensure convergence of GA, the optimum individual of each generation is reserved to the next generation. Breakpoint crossover and exchange mutation can be applied in crossover and mutation process.

\section{Chromosome rehabilitation}

Considering capacity constraint of distribution centers, the number of subcenters linked to each center can not exceed $g$, therefore, there may be population that can not satisfy this constraint in crossover and mutation process. If we attempt to regenerate a new population of this kind, it will waste time and resources. Therefore, rehabilitation needs to be implemented on links between distribution center and subcenters. Let $G$ denote a set of distribution centers that satisfy the above constraints. If the number of subcenters linked to center $i$ exceeds $g$, substitute $i$ with a random selection from $G$.

\section{Terminal conditions}

Terminate search when any one of the following conditions is satisfied:

1) Predefine a maximum number of generation times and terminate until iterative times reach the maximum number;

2) Overall satisfaction degree will not change any more or change little during the span of a number of generation times.

\section{NUMERICAL EXPERIMENT RESULTS}

Define parameters of GA as follows: population size $=80$, maximun iterative times $=100$, crossover rate $=0.6$, mutation rate $=0.2$. For satisfaction function of transportation cost, let $a_{1}=400, a_{2}=900$; for satisfaction function of average delay, let $b_{1}=5, b_{2}=50$; for satisfaction function of capacity reliability, let $c_{1}=0.6, c_{2}=$ $0.80, c_{3}=1.00$. In addition, let $a=0.5, b=0.6, c=0.8$.

Considering transportation cost, average delay and capacity reliability as equally important, let $w_{c}=w_{r}=w_{d}=$ $1 / 3$ in calculation of overall satisfaction function. Then, the optimization results are shown as Table 1 and the corresponding logistics network structure is shown as Figure 6, and the curve of overall satisfaction degree in the GA search process is shown as Figure 7.

Figure 7 showed that overall satisfaction degree enhances with generation number of GA search, and finally, kept unchanged. The convergence speed was fast that demonstrates the validity of the multi-objective satisfactory optimization method for logistics network.

\section{DISCUSSION}

The optimization result of the numerical example showed its effectiveness and efficiency. The proposed approach 
Table 1. Performance indexes and optimization results.

\begin{tabular}{cccccccc}
\hline Weight & $\begin{array}{c}\text { Transportation } \\
\text { cost }\end{array}$ & $\begin{array}{c}\text { Average } \\
\text { delay }\end{array}$ & $\begin{array}{c}\text { Capacity } \\
\text { reliability }\end{array}$ & $\begin{array}{c}\text { Satisfaction } \\
\text { degree of } \\
\text { transportation } \\
\text { cost }\end{array}$ & $\begin{array}{c}\text { Satisfaction } \\
\text { degree of } \\
\text { average } \\
\text { delay }\end{array}$ & $\begin{array}{c}\text { Satisfaction } \\
\text { degree of } \\
\text { capacity } \\
\text { reliability }\end{array}$ & $\begin{array}{c}\text { Overall } \\
\text { satisfaction } \\
\text { degree }\end{array}$ \\
\hline $\mathrm{W}_{\mathrm{c}}=\mathrm{W}_{\mathrm{d}}=\mathrm{W}_{\mathrm{r}}=1 / 3$ & 582 & 27 & 0.92 & 0.82 & 0.80 & 0.92 & 0.85 \\
\hline
\end{tabular}

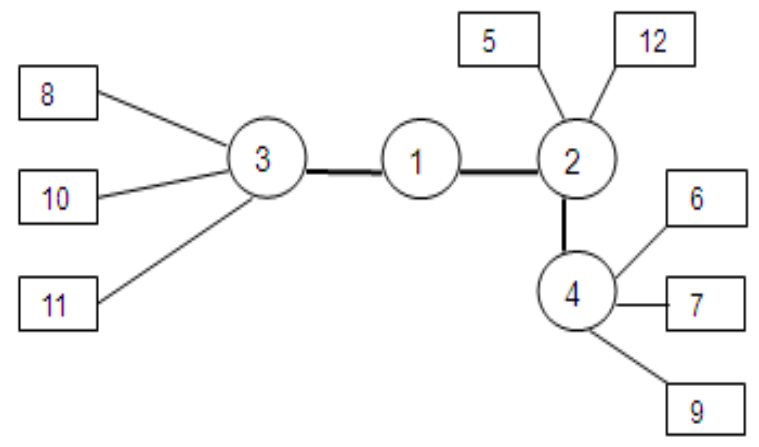

Distribution center Distribution subcenter

Figure 6. Optimal logistics network structure.

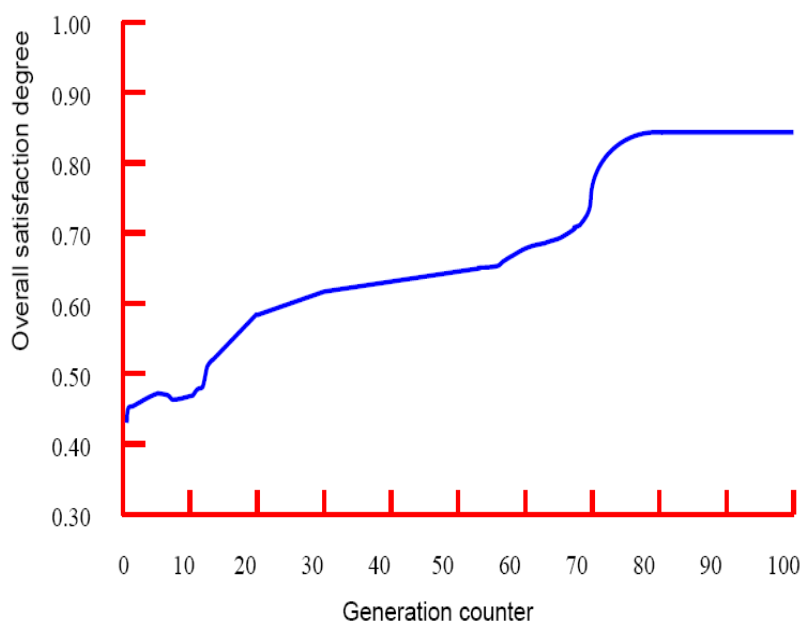

Figure 7. Curve of overall satisfaction degree.

exhibited the potential applicability and significance of multi-objective satisfactory optimization techniques on the design of logistics network. The presented methodology is of great significance, especially when the objective function incorporates conflicting variables, for practical applications in the field of logistics network design. The feature of multi-objective satisfactory optimization is of high significance equally to both decision makers as well as the analysts because the analysts are concerned with fulfilling multiple objectives simultaneously while decision makers are concerned with the quality of their decisions (Singh and Chakrabarty, 2010). The intentions of both parties can be accomplished by adopting the presented methodology. The proposed model could be useful in solving a wide variety of similar problems, and help in making better decisions in transportation and logistics systems with multiple objectives. The application of multiobjective satisfactory optimization method is not limited in system design, and this paper only adopted network structure as an example to demonstrate the applicability of this method. Some detailed multi-level considerations can also be tackled in similar ways.

\section{CONCLUSIONS}

This research provides a new insight for assessment of capacity reliability of road network and offers a new perspective for logistics network optimization with reliability consideration. The intrinsic similarity between traffic flow and liquid flow may help researcher in transportation science to discover some law for further development of the theory and practice of transportation engineering and management. Large-scale logistics network optimization is a complicated and difficult task, and essentially, a multi-objective satisfactory optimization problem. This practical approach presented in this paper can solve some similar problems in logistics system and contributed to management method and operation practice. It allows a broader view of the problem and weights the alternatives in accordance with managers' inclination.

\section{ACKNOWLEDGEMENTS}

This work is supported by Humanities and Social Science Fund of the Ministry of Education of China (09YJC630049), Natural Scientific Research Innovation Foundation in Harbin Institute of Technology (HIT.NSFIR.2010082), National Natural Science Foundation of China (61074133), Natural Science Foundation of Heilongjiang Province (G201014), China Postdoctoral Science Foundation funded project (20090450125, 201003432), Heilongjiang Postdoctoral Fund (LBH-Z09114), and 2010 Humanities and Social 
Science Guidance Programme of Education Department of Heilongjiang Province (11554097). The authors sincerely thank the anonymous reviewers and the relevant editorial board members for their valuable comments and suggestions.

\section{REFERENCES}

Akgungor AP, Dogan E (2009). An application of modified Smeed, adapted Andreassen and artificial neural network accident models to three metropolitan cities of Turkey. Sci. Res. Essays., 4(9): 906-913.

Amorocho J, Espildor B (1973). Entropy in assessment of uncertainty in hydrologic systems and models. Water Resour. Res., 9(6): 15111522.

Ang WK, Jowitt PW (2005a). Some new insights on informational entropy for water distribution networks. Eng. Optimiz., 37(3): 277289.

Ang WK, Jowitt PW (2005b). Path entropy method for multiple-source water distribution networks. Eng. Optimiz., 37(7): 705-715.

Awumah K, Goulter I, Bhatt SK (1990). Assessment of reliability in water distribution networks using entropy-based measures. Stoch. Hydrol. Hydraul., 4(4): 325-336.

Awumah K, Goulter I, Bhatt SK (1991). Entropy-based redundancy measures in water distribution network design. ASCE J. Hydr. Eng., 117(5): 595-614.

Badri MA, Mortagy AK, Alsayed A. (1998). A multi-objective model for locating fire stations. Eur. J. Oper. Res., 110 (2): 243-260.

Barnhart C, Shen S (2005). Logistics service network design for timecritical delivery. Lect. Notes. Comput. Sc., 3616: 86-105.

Bialynickibirula I, Mycielski J (1975). Uncertainty relations for information entropy in wave mechanics. Commun. Math. Phys., 44(2): 129-132.

Candas MF, Kutanoglu E (2007). Benefits of considering inventory in service parts logistics network design problems with time-based service constraints. IIE Trans., 39(2): 159-176.

Castans M (1962). Entropy and Uncertainty. Am. J. Phys., 30(7): 521527.

Chen A, Yang H, Lo HK, Tang WH (1999). A capacity related reliability for transportation networks. J. Adv. Transport., 33(2): 183-200.

Chen A, Yang H, Lo HK, Tang WH (2002). Capacity reliability of a road network: An assessment methodology and numerical results. Transport. Res. B-Meth., 36(3): 225-252.

Cheong MLF, Bhatnagar R, Graves SC (2007). Logistics network design with supplier consolidation hubs and multiple shipment options. J. Ind. Manage. Optim., 3(1): 51-69.

Chiou SW (2009). A bi-level programming for logistics network design with system-optimized flows. Inf. Sci., 179(14): 2434-2441.

Cordeau JF, Laporte G, Pasin F (2008). An iterated local search heuristic for the logistics network design problem with single assignment. Int. J. Prod. Econ., 113(2): 626-640.

Cordeau JF, Pasin F, Solomon MM. (2006). An integrated model for logistics network design. Ann. Oper. Res., 144(1): 59-82.

da Silva AML, Fernandez RAG, Singh C (2010). Generating capacity reliability evaluation based on Monte Carlo simulation and crossentropy methods. IEEE T. Power Syst., 25(1): 129-137.

Di Crescenzo A, Longobardi M (2002). Entropy-based measure of uncertainty in past lifetime distributions. J. Appl. Probab., 39(2): 434440.

Eskigun E, Uzsoy R, Preckel PV, Beaujon G, Krishnan S, Tew JD (2005). Outbound supply chain network design with mode selection, lead times and capacitated vehicle distribution centers - Production, manufacturing and logistics. Eur. J. Oper. Res., 165(1): 182-206.

Gundogdu IB (2009). Spatial analyst methods for urban planning. Sci. Res. Essays., 4(12): 1531-1535.

Gursoy M (2010). A method for transportation mode choice. Sci. Res. Essays. 5(7): 613-624.

Ho W, Emrouznejad A (2009). Multi-criteria logistics distribution network design using SAS/OR. Expert. Syst. Appl., 36(3): 7288-7298.
Huang HZ, Tao Y, Liu Y (2008). Multidisciplinary collaborative optimization using fuzzy satisfaction degree and fuzzy sufficiency degree model. Soft. Comput., 12(10): 995-1005.

Jaynes ET (1957). Information theory and statistical mechanics. Phys. Rev., 106(4): 620-630.

Jeet V, Kutanoglu E, Partani A (2009). Logistics network design with inventory stocking for low-demand parts: Modeling and optimization. IIE Trans., 41(5): 389-407.

Johansson M, Sternad M (2005). Resource allocation under uncertainty using the maximum entropy principle. IEEE T. Inform. Theory., 51(12): 4103-4117.

Kofteci S, Ergun M, Ay HS (2010). Modeling freight transportation preferences: Conjoint analysis for Turkish Region. Sci. Res. Essays., 5(15): 2016-2021.

Li SY, Hu CF (2007). Two-step interactive satisfactory method for fuzzy multiple objective optimization with preemptive priorities. IEEE T. Fuzzy. Syst., 15(3): 417-425.

Li XB (2009). Study of multi-objective optimization and multi-attribute decision-making for economic and environmental power dispatch. Electr. Pow. Syst. Res., 79(5): 789-795.

Lighthill MJ, Whitham JB (1955). On kinetic waves. I: Flow movement in long rivers. II: A theory of traffic flow on long crowded roads. P Roy Soc A-Math Phy., 229: 281-345.

Liu GSA (2006). Combination method for reliability-redundancy optimization. Eng. Optimiz., 38(4): 485-499.

Liu SB, Cheng KH, Liu XP (2000). Network reliability with node failures. Networks. 35(2): 109-117.

Malakooti B (1989). Multiple objective facility layout: a heuristic to generate efficient alternatives. Int. J. Prod. Res., 27(7): 1225-1238.

Miao X, Xi B, Zhang XQ (2008). Effect equilibrium approach in calculating the economic range of a freeway industrial zone. Eur. J. Transport. Infrastruct. Res., 8(1): 31-43.

Miao X, Yu B, Xi B (2009). The uncertainty evaluation method of supply chain reliability. Transport., 24(4): 296-300.

Ok SY, Song J, Park KS (2009). Development of optimal design formula for bi-tuned mass dampers using multi-objective optimization. $J$ Sound. Vib., 322(1-2): 60-77.

Rajagopal AK, Teitler S (1989). Minimum uncertainty products from the principle of maximum-entropy. Phys. Rev. A., 40(2): 515-518.

Schott JP, Mcclellan JH (1984). Maximum-entropy power spectrum estimation with uncertainty in correlation-measurements. IEEE T. Acoust. Speech Signal Proces., 32(2): 410-418.

Shannon CE (1948). A mathematical theory of communication. Bell Syst. Tech. J., 27(3): 379-428.

Singh TS, Chakrabarty D (2010). Multi-objective optimization for optimal groundwater remediation design and management systems. Geosci. J., 14(1): 87-97.

Sreekumar, Mahapatra SS (2009). A fuzzy multi-criteria decision making approach for supplier selection in supply chain management. Afr. J. Bus. Manage., 3(4): 168-177.

Sumalee A, Kurauchi $F$ (2006). Network capacity reliability analysis considering traffic regulation after a major disaster. Netw. Spat. Econ., 6(3-4): 205-219.

Tang YH, Xi B (2010). Dynamic forecasting of traffic volume based on quantificational dynamics: A nearness perspective. Sci. Res. Essays., 5(4): 389-394.

Tanyimboh TT, Templeman AB (1993a). Calculating maximum entropy flows in networks. J. Oper. Res. Soc., 44(4): 383-393.

Tanyimboh TT, Templeman AB (1993b). Maximum entropy flows for single-source networks. Eng. Optimiz., 22(1): 49-63.

Tanyimboh TT, Templeman AB (2000). Aquantified assessment of the relationship between the reliability and entropy of water distribution systems. Eng. Optimiz., 33(2): 179-199.

Wen BL, Tan XH, Sun HL, Li YM (2006). Flow and capacity distribution of computer communication networks based on multi-criteria satisfactory optimization. Comput. Appl., 26(12): 2806-2812.

Wu YB, Tian H (2003). Entropy-based water distribution network rehabilitation. International Conference on Advances in Water Supply Management. London, England, pp. 15-17. 